\title{
APRENDIZAJE EN EL PROCESO DE INCUBACIÓN DE EMPRESAS DE BASE TECNOLÓGICA'
}

\author{
Anne-Marie Maculan² - Claudia Nelcy Jiménez Hernández ${ }^{3}$. \\ Oscar Fernando Castellanos Domínguez ${ }^{4}$
}

Universidad Nacional de Colombia, Colombia - Universidad Federal de Rio de Janeiro, Brasil - Artículo Tipo 1. Investigación Científica y Tecnológica - Recibido: 20 de Enero 2015 - Aceptado: 20 de Junio 2015

\section{RESUMEN}

El estudio de los procesos de aprendizaje, permite evidenciar los factores que influyen en la capacidad de gestión de innovaciones tecnológicas y organizacionales, así como en los posibles lucros de competitividad de la economía en su conjunto. El objetivo de este artículo, es analizar este proceso como experiencia de las empresas nacientes en incubadoras de base tecnológica, principalmente universitarias. Para ello, se revisaron varios estudios sobre el tema, con el fin de discutir el rol de la tecnología y el conocimiento en las Empresas de Base Tecnológica (EBT), y la experiencia brasileña como país líder en Latinoamérica en la creación de estas mismas mediante incubadoras universitarias. Esto permitió plantear consideraciones sobre los rasgos e importancia de la incubación en el aprendizaje y generación de capacidades de las EBT, como la necesidad de que las incubadoras faciliten su inserción en redes de información, producción y servicios, así como en los sistemas nacionales y regionales de innovación. Se concluyó que el éxito de estas empresas depende en gran medida del capital relacional de la incubadora, y que el papel de esta última resulta clave en su transición desde la categoría de empresas tecnicistas a empresas inteligentes.

\section{Palabras Clave:}

Incubación de Empresas, Innovación e invención, Gestión de tecnología.

JEL: M13, O31, M15

\section{Si va a referenciar este artículo}

Maculan, A., Jimenez, C. \& Castellanos, O. (2015). Aprendizaje en el proceso de incubación de empresas de base tecnológica, Económicas CUC, 36 (1), 9-26

\footnotetext{
${ }^{1}$ Artículo de reflexión que retoma resultados de la investigación "From research to innovation: the Brazilian experience with business incubators" y alimentado con las investigaciones, "Ambiente emprendedor e aprendizado das pequeñas empresas de base tecnológica", "Empresas graduadas más incubadoras brasileiras", "Incidencia del nivel de asimilación del conocimiento y la tecnología en el desarrollo empresarial", "Consideraciones metodológicas para el análisis de la competitividad en empresas de base tecnológica", financiados por la Universidad Federal de Rio de Janeiro en Brasil y Universidad Nacional de Colombia.

${ }^{2}$ Ph.D. en Socio-Economía, Université du Québec a Montreal (UQAM), Canadá. Profesora del Programa de Ingeniería de Producción del Instituto Alberto Luiz Coimbra de Pós-Graduação e Pesquisa de Engenharia COPPE - Universidade Federal do Rio de Janeiro, Brasil. amaculan@pep.ufrj.br

${ }^{3}$ Magíster en Administración, Candidata a Ph.D. en Ingeniería, Universidad Nacional de Colombia. Profesora Facultad de Ciencias Agrarias, Universidad Nacional de Colombia. Investigadora del Grupo Biogestión. cnjimenezh@unal.edu.co

${ }^{4}$ Ph.D. en Ciencias, Universidad Estatal de Moscú Lomonosov, Rusia. Profesor Facultad de Ingeniería, Universidad Nacional de Colombia. Director del Grupo de investigación y desarrollo en gestión, productividad y competitividad Biogestión. ofcastellanosd@unal.edu.co
} 


\section{INTRODUCCIÓN}

En los últimos años, algunos países han experimentado el surgimiento cada vez mayor de pequeñas empresas de base tecnológica fundadas en las incubadoras, que están transformándose en objeto de análisis debido a que están desarrollando y difundiendo nuevas modalidades de organización en las actividades productivas. Nacen frecuentemente unidas a un proceso de reformulación de las relaciones entre universidades o instituciones de Investigación y Desarrollo (I+D) y empresas; por tanto, están vinculadas a un nuevo marco de transferencia de conocimientos de la comunidad académica hacia la industria.

Mustar, Clarysse \& Wright (2007), afirman que la transferencia de tecnología y conocimiento desde las universidades hacia la industria, tradicionalmente ha tenido énfasis en el licenciamiento de innovaciones, pero internacionalmente la atención ha comenzado a orientarse a la creación de nuevos negocios basados en los desarrollos de las organizaciones de investigación pública; este es un fenómeno relacionado con un conjunto de cuestiones de extrema importancia:

- El fomento al espíritu innovador.

- El cambio del comportamiento innovador de las pequeñas empresas.

- La dinámica del desarrollo local y regional.

- La formación de una cultura productiva local.

- Las complejas relaciones entre ciencia e industria.

- Las nuevas formas que puede adoptar la valorización del saber académico.

- La capacidad de explorar nichos de mercado aún no identificados.
En este sentido, Rasmussen \& Borch (2010), señalan que la habilidad de las universidades y centros de I+D para facilitar la iniciación de actividades emprendedoras, que se centran en la identificación y explotación de oportunidades, es un elemento clave en la creación de empresas de base tecnológica. A través del estudio de estas experiencias se podrá avanzar en el conocimiento sobre las condiciones de creación y de permanencia de este tipo de empresas en el mercado y de manera más amplia, del dinamismo del sistema industrial.

Un aspecto fundamental en este análisis es el aprendizaje, como un proceso continuo y dinámico que lleva a que las organizaciones modifiquen o adopten acciones mediante la adquisición y asimilación de conocimiento sostenible (Hung, Lien, Yang, Wu, \& Kuo, 2011); además, determina la competitividad al contribuir a una mejor y veloz adquisición y explotación de la información del mercado en comparación con los competidores. (Jiménez \& Sanz, 2006)

El objetivo de este artículo es analizar el proceso de aprendizaje de las empresas de base tecnológica nacidas en las incubadoras, particularmente las universitarias, enfatizando la importancia de la experiencia de incubación para la adquisición de capacidades tecnológicas y gerenciales. Inicialmente se presenta el concepto de aprendizaje, luego se discute sobre el papel de la tecnología y el conocimiento en las EBT. Más adelante, tomando a Brasil como ejemplo de análisis, por tratarse de un país líder en Latinoamérica en este tipo de dinámicas, se revisan los rasgos de las EBT incubadas como aporte a la comprensión del papel de estas empresas en medio de las grandes y recientes transformaciones de la economía brasileña, finalizando con algunas consideraciones sobre cómo aprenden las empresas durante los procesos de incubación. 


\section{Aprendizaje organizacional}

El aprendizaje organizacional es un proceso en el que el conocimiento se adquiere del exterior o es generado internamente en la empresa, se distribuye a lo largo de la misma, se interioriza y se le busca un significado común y finalmente se almacena en la memoria organizativa Huber (citado por Jiménez \& Sanz, 2006). Exige la disposición de recursos y la definición de métodos y procedimientos para registrar y codificar los conocimientos tácitos generados por las experiencias; siendo necesario que la empresa cree competencias organizacionales para asimilar este tipo de informaciones. Este abordaje se centra en la dimensión cognitiva de la firma como organización capaz de tratar las informaciones, ampliar y valorizar los conocimientos acumulados a partir de fuentes internas y externas. (Maculan, 2003)

\section{El aprendizaje como un proceso interno y dependiente de la trayectoria: Visión Evolutiva}

La naturaleza del proceso evolutivo es definida por Nelson (2003), en términos conductuales que explican las mejoras graduales mediante procesos de aprendizaje individual y colectivo; se habla entonces de procesos en los cuales los individuos y las firmas aprenden y mejoran en el tiempo, que son dependientes de la trayectoria puesto que existirán influencias del contexto y de las condiciones particulares en los que ocurran dichos procesos, de manera que no habrá dos resultados iguales.

Estrada (2005), enfatiza dos postulados evolucionistas, por ser los que más han influido en el avance de la economía de la innovación: concebir a la tecnología como conocimiento, no como información y considerar el aprendizaje heterogéneo entre los agentes. Estos postulados llevan a que, como conocimiento, la tecnología no sea transferida fácilmente ni apropiada por completo, en tanto su aprendizaje es específico para cada empresa. Para la teoría evolutiva estas diferencias entre las empresas son inevitables y fundamentales.

En la teoría evolucionista, el aprendizaje es un concepto clave como proceso interno de acumulación de experiencias y habilidades específicas de cada empresa, ligadas a su capacidad de producción. Por medio de la experimentación y de la repetición, la empresa consigue mejorar la ejecución de sus diferentes tareas y explorar rápidamente nuevas oportunidades. El aprendizaje se cristaliza con la búsqueda de soluciones para organizar la producción, tanto en el entrenamiento de recursos humanos como en la adopción de nuevas prácticas gerenciales y organizacionales; este aprendizaje es acumulativo y colectivo, e implica la adquisición de competencias tanto por los individuos como por las organizaciones. Este proceso ocurre según las reglas específicas de cada empresa, y presupone que la organización destina recursos con esa finalidad; en otras palabras, exige la definición de métodos y objetivos. Bell (1984); Katz (1984)

El recordar haciendo (remember by doing), es un concepto propuesto por Nelson \& Winter (1982), como medio a través del cual la organización almacena un conocimiento operacional específico en lo que se podría denominar la memoria organizacional, es decir, convertir en rutina una actividad; el aprendizaje a través del remember by doing agrega conocimientos esencialmente de naturaleza tácita, que están ligados a las prácticas y son gradualmente transformados en rutinas $\mathrm{y}$ repertorios de procedimientos. Sin embargo, el aprendizaje también puede ocurrir a partir de las actividades altamente formalizadas de generación interna de conocimientos como las actividades de $\mathrm{I}+\mathrm{D}$, que Viotti (2002), denomina non-doing-based 
learning o innovación incremental activa, consecuencia de esfuerzos e inversiones deliberadas en tecnología.

Por otro lado, el aprendizaje corresponde a la asimilación de conocimientos de origen externo, obtenidos por las interacciones con otras empresas en el mercado, dentro de las relaciones productor-usuario, o con fuentes institucionales de investigación o de entrenamiento, laboratorios especializados, empresas que proporcionan equipos o insumos, compradores, consultores, agencias de gobierno, etc. Para aprovechar los conocimientos de fuentes externas es necesario establecer interacciones permanentes y regulares con otras organizaciones y disponer de habilidades internas para asimilar esos conocimientos e incorporarlos como soluciones a necesidades específicas de cada empresa. En este tipo de aprendizaje es evidente la dimensión estratégica de las interacciones.

La necesidad de multiplicar las interacciones conduce a algunas empresas a implementar una dinámica de cooperación y de aproximación en las relaciones con universidades o con otras empresas. Algunas instituciones cuya finalidad es la producción de conocimientos universales y codificados - escuelas técnicas, centros de I+D, grandes programas públicos de apoyo a los estudios pre-competitivos - privilegian ciertas maneras de creación y difusión de conocimientos no exclusivas y establecen múltiples formas de cooperación. A pesar de ello, la difusión de conocimientos continúa ocurriendo de manera muy informal, con base en contactos entre individuos, resultando difícil su identificación y medición.

Otra importante dimensión del aprendizaje es la necesidad de creación de rutinas organizacionales que incorporen el conocimiento. Debido a la existencia de límites cognoscitivos en los individuos y a la com- plejidad de sus interacciones, la empresa aprende solamente si ella misma crea una capacidad interna de incorporación de los conocimientos tácitos en rutinas y procedimientos, para socializarlos dentro de la organización, documentando el conocimiento distintivo que puede ser codificado (Dutrénit, 2001). Los procedimientos son el resultado de aprendizaje consolidado que agrupa los conocimientos tácitos, y expresan la importancia de la dimensión organizacional del aprendizaje, constituyendo un repertorio de respuestas a partir del saber individual y colectivo, de las experiencias y habilidades, que actúa de forma automática.

Sin embargo, para las empresas representa una dificultad registrar los conocimientos tácitos, no formalizados, para incorporarlos en rutinas que necesitarán un cierto grado de formalización, con el fin de que sean eficientes. Además, el grado de dinamismo de las rutinas organizacionales es diferente según el tipo de interacción y son meramente repetitivas y estáticas, en cuanto otras (comportamientos más arriesgados de búsqueda, ensayo y error que objetivan el desarrollo de innovaciones) están destinadas a promover la adquisición y la acumulación de nuevos conocimientos.

\section{Obstáculos al aprendizaje en las organizaciones}

El aprendizaje puede verse obstaculizado por la falta de información sobre las fuentes institucionales de conocimientos, debido a la multiplicidad o escasez de esas fuentes, por la distancia organizacional o por la existencia de los derechos de propiedad exclusiva (privada); sin embargo, el aprendizaje también puede surgir por las múltiples interacciones entre organizaciones económicas y otras instituciones, el cual se conoce como aprendizaje por interacción (learning by interacting). Enton- 
ces, ¿cuál será el espacio en el que ha de realizarse en forma más intensa este learning by interacting? Para responder esta pregunta, Lundvall (1988), utiliza el concepto de Sistema Nacional de Innovación (SNI), el cual ofrece la posibilidad de integrar las múltiples fuentes de aprendizaje dentro de un contexto amplio, y de poner en evidencia esta dimensión institucional. Si bien el proceso de globalización conduce a uniformizar los modos de producción, el concepto de SNI insiste sobre la prominencia de la dimensión nacional o local en las interacciones de los actores.

Para comprender el proceso de aprendizaje y de capacitación de las pequeñas empresas es importante analizar su dinámica de inserción en el sistema local de innovación y en las redes de subcontratación, así como su localización en incubadoras. Es en este contexto que ellas aprenden y se insertan en el mercado. (Cooke, Gomez, \& Etxebarria, 1997)

Viotti (2002), señala que el marco conceptual y teórico de SNI no es apropiado para el caso de los procesos de cambio técnico típicos de economías en vías de industrialización, ya que se emplea el concepto tradicional de innovación en sentido estricto, el cual está relacionado directamente con la capacidad de las empresas para realizar actividades de I+D e innovación $(\mathrm{I}+\mathrm{D}+\mathrm{I})$, que es de baja o nula ocurrencia en dichas economías. Adicionalmente, algunos SNI son propensos a seguir la estrategia tecnológica dirigida solamente a la absorción de capacidades de producción, por lo cual son considerados como sistemas pasivos; Viotti analizó el caso de Brasil como representativo de esta situación. Entre tanto, algunos países han seguido una estrategia de aprendizaje activo, que implica el dominio y mejoramiento de las tecnologías de producción absorbidas, característica de economías con SNI activos, como es el caso de Corea.
Arocena \& Sutz (2006), afirman que la innovación realmente existente en los países en desarrollo es de carácter altamente informal, pero no por ello puede llegarse a la falsa conclusión de que prácticamente no existe; empero, estos autores son categóricos en afirmar que en entornos atrasados no se puede dar por supuesto que la innovación tenga carácter sistémico:

Se realiza, por cierto, a través de vínculos e interacciones entre actores diversos, pero unos y otras suelen ser frágiles, episódicos y escasos. Los Sistemas de Innovación son más potenciales que reales. Esto tiene importancia teórica, pero sobre todo práctica: las políticas para la innovación en el Subdesarrollo no pueden dar por sentado que los «sistemas» existen y funcionan como tales. (Arocena \& Sutz, 2006, sección La innovación desde el Sur, párr. 5).

\section{Conocimiento y tecnología en los procesos de aprendizaje de las empresas de base tecnológica}

Las Empresas de Base Tecnológica (EBT), son el resultado de la etapa más reciente del desarrollo industrial y cada vez tienen mayor impacto económico en relación con la relevancia de la tecnología y el conocimiento en los procesos de producción. En cuanto a la generación y consolidación de EBT, y con el imperativo de trascender el ámbito de la I+D e involucrarse en mayor medida con otras áreas de la organización y de su entorno, la gestión tecnológica busca generar marcos conceptuales, metodologías y herramientas que fortalezcan su papel frente a la tecnología como pilar de la competitividad y a la consolidación de la sociedad del conocimiento. (Jiménez \& Castellanos, 2008)

Las EBT son elementos clave de estructuración de los Sistemas Nacionales de Innovación, por lo cual requieren ser estudiadas para conocer mejor sus peculiaridades, 
partiendo de la premisa de que la gestión de la tecnología y el conocimiento influye en las organizaciones al favorecer procesos de transferencia tecnológica y de conocimiento que conlleven al aprendizaje y por tanto, impactan en la competitividad, en especial cuando se hace referencia a este nuevo tipo de empresa denominada de base tecnológica.

\section{Características de las EBT}

Estas empresas se basan en el dominio y explotación intensiva del conocimiento científico y técnico (Simón, 2003). Chamanski \& Waagø (2001), afirman que las EBT aportan a la sociedad nuevos productos, servicios o tecnologías que incrementan la eficiencia de las industrias al reducir los costos de transacción por adquisición de tecnología, mejorando sus procesos de desarrollo y generando nuevos campos de innovación.

La EBT es un concepto transversal que no se refiere únicamente a su resultado final que es un producto de alto valor agregado o de alta complejidad tecnológica, sino que inicia desde la incorporación de conocimiento como materia prima fundamental para el logro de sus objetivos, hasta su posterior transformación en el valor de la línea principal de un producto concreto (Incubadora de Empresas de Base Tecnológica de Antioquia - IEBTA, 2006). La importancia de las EBT radica en que sustentan sus estrategias de gestión y su línea de procesos, productos y servicios en nuevas tecnologías e involucran los desarrollos administrativos, gerenciales, económicos, financieros, de capacitación e $\mathrm{I}+\mathrm{D}$ de última generación a sus operaciones (Morales, Castellanos, \& Jiménez, 2007).

Las Empresas de Base Tecnológica tienen dos componentes específicos que las identifican (Carvalho, Machado, Pisysieznig-Filho, \& Rabechini-Junior, 1998): (1) generalmente son pequeñas o medianas, ocupando poco personal altamente calificado y (2) producen bienes y servicios con alto valor agregado a partir del conocimiento; además, tienden a relacionarse con las universidades, institutos o centros de investigación, donde se generan tecnologías en áreas de conocimiento similares a las que requieren para su desarrollo y actualización tecnológica.

En muchas ocasiones las EBT se originan en estas organizaciones de investigación o tienden a localizarse en sus cercanías; un caso particular de estas son las Spin-offs ${ }^{5}$, que se crean al interior de una organización mayor, generalmente una universidad, por iniciativa de algún investigador de la misma institución y se basan en la trasferencia de tecnología y de personal; la universidad cumple la función de incubadora, mientras que sus fundadores son profesores, alumnos o miembros del personal administrativo, que cuentan con apoyo institucional (Morales, 2009; Universidad de Barcelona, 2010). La Spin-off es un mecanismo de transferencia de los desarrollos tecnológicos de una institución de I+D o una universidad. (Clarysse \& Moray, 2004)

Según Castellanos (2007), al contrario de organizaciones tradicionales, el aprendizaje en las EBT no recae en un selecto grupo de personas encargadas de estos temas, sino que se forma como un proceso colectivo de la organización, donde el aprendizaje es una tarea constante a cargo de todos los miembros de la empresa, teniendo como objetivo compartir y sobre todo, crear conocimiento, las EBT se reconocen por su búsqueda permanente de innovaciones y por su capacidad para buscar información, generar e integrar nuevo conocimiento.

\footnotetext{
5 Es un término anglosajón que se refiere a un proyecto nacido como extensión de otro anterior, o más aún de una empresa nacida a partir de otra mediante la separación de una división subsidiaria o departamento de la empresa para convertirse en una empresa por sí misma.
} 


\section{Categorías de EBT según el impacto de la tecnología y el conocimiento}

Si bien la tecnología es parte fundamental de los procesos productivos, no todas las empresas aprovechan y asimilan el factor tecnológico de igual manera, o no disponen de tecnologías modernas y recurren a aquellas de dominio público o en la obsolescencia. Esto puede estar relacionado con la capacidad gerencial y la disposición para aprender de sus integrantes. En este sentido, Granados, Castellanos \& León (2006), proponen un modelo para el análisis de la incidencia de la tecnología y el conocimiento en las organizaciones, considerando diferentes dimensiones de estos dos factores: en el caso del conocimiento, se establecen dos categorías sustentadas en el aprendizaje, como una de sus características más relevantes: organizaciones que aprenden y organizaciones que no aprenden. Respecto a la tecnología, se hace referencia a empresas de base tecnológica y empresas con precaria base tecnológica.
Así, estos autores identifican cuatro tipos de empresas: tradicionales, fraternalistas, tecnicistas e inteligentes. Ver Figura 1.

Las empresas con precaria base tecnológica que no aprenden, es decir, que no tienen capacidades internas para buscar y generar nuevos conocimientos y llamadas tradicionales, se caracterizan por su baja competitividad; al no tener procesos de gestión tecnológica y del conocimiento, su cadena de valor es bastante frágil y su valor agregado es bajo, nulo o negativo.

Por otra parte, las empresas fraternalistas, es decir, empresas con precaria base tecnológica que aprenden, se identifican porque saben usar el conocimiento como un insumo de su actividad productiva, lo dominan y tienen capacidades dinámicas de aprendizaje, pero no cuentan con los medios para aprovecharlo efectivamente y por tanto, deben limitarse al desarrollo de procesos y productos compatibles con las herramientas productivas que poseen, asociadas a restricciones en su capacidad financiera

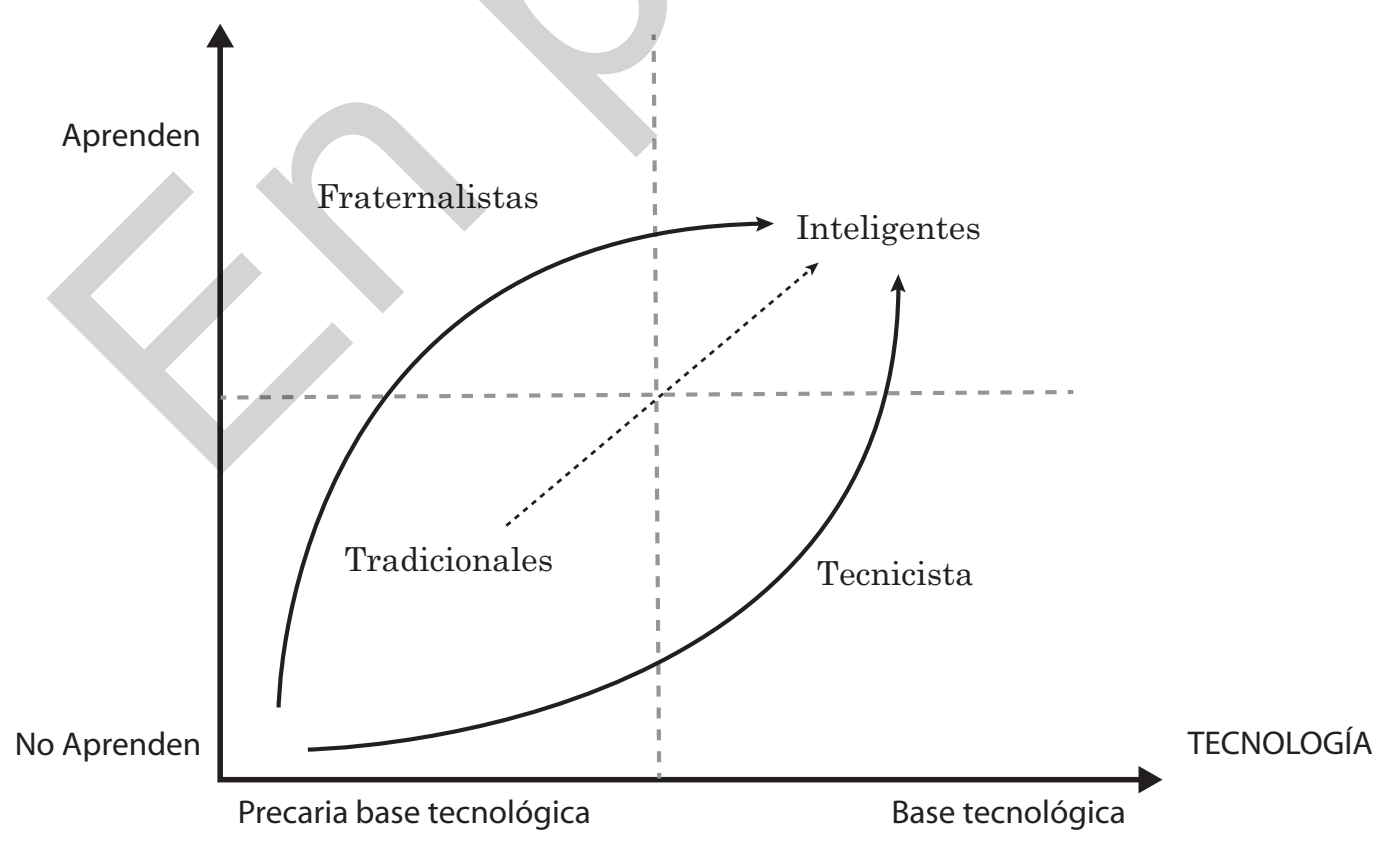

Figura 1. Incidencia del conocimiento y la tecnología en el desarrollo empresarial 
o de mercado, o a un contexto de tecnologías escasas, atrasadas y que no promueve la adquisición o generación de tecnologías. Así, el valor que estas empresas pueden llegar a ofrecer en sus productos se ve notablemente disminuido con el riesgo de quedarse rezagadas en el mercado. Este tipo de empresas resultaría muy beneficiado con la existencia de políticas públicas que favorezcan los procesos de aprendizaje.

Las empresas Tecnicistas son empresas de base tecnológica que no logran aprender y evolucionar, porque sus procesos de gestión de conocimiento fallan al no ser colectivos y no crear conocimiento organizacional. Aunque la firma, a través de sus integrantes, domine y comprenda el conocimiento y la tecnología que posee, no tiene procesos de generación de conocimiento definidos, por lo que su cadena de valor se afecta y su competitividad no es elevada. El rasgo principal es que están integradas por personal altamente calificado, generalmente investigadores y expertos en un campo particular, que no comparten sus conocimientos ni participan en dinámicas de trabajo en equipo.

Los activos intangibles de la empresa son bajos y están representados principalmente por sus integrantes (capital humano), mientras que el capital relacional y el capital estructural presentan notables deficiencias y en algunos casos no existen. No obstante, estas empresas se encuentran con dificultades para acceder a tecnologías más avanzadas que en general pertenecen a sus competidores, o pueden estar en mercados poco dinámicos que las obstaculizan.

Las empresas Inteligentes son de base tecnológica y al mismo tiempo son empresas que aprenden, con lo que pueden garantizar esquemas de generación de conocimiento, innovación y de construcción de relaciones mucho más complejas entre todos sus procesos y con su contexto.
Estas organizaciones diseminan el conocimiento, modificando su arquitectura de toma de decisiones para que las personas con la mejor perspectiva las tomen, alineando sus actividades con la estrategia y con estructuras organizacionales en red para innovar continuamente. De esta manera aumentan no solo su valor, sino su competitividad al ofrecer características nuevas y únicas que tengan acogida en la cadena de valor de sus clientes. Su énfasis en la generación de conocimiento traducido a productos o servicios se ve reflejado en el aumento del valor de la compañía, ya que tendrán un mayor volumen de activos intangibles en todas sus áreas.

Finalmente, al conjugar procesos de gestión de conocimiento y de gestión tecnológica, este tipo de empresas consigue construir competencias estratégicas que perduran y evolucionan en el tiempo, por lo que son difíciles de imitar y pueden transformar los conocimientos en éxitos comerciales. Las empresas inteligentes son resultado de varios años de actuación en el mercado y de considerables inversiones en investigación, conocimiento, formación de recursos humanos y estrechas relaciones con las empresas usuarias de sus productos y servicios.

Tomando en cuenta la categorización descrita por Granados et al. (2006), puede afirmarse que en general, las EBT de contextos de menor desarrollo se ubican en las categorías fraternalista y tecnicista, pero mediante políticas públicas, programas de apoyo y articulación con los demás actores del sistema de innovación local y nacional, pueden abrirse espacios para su fortalecimiento tendiente a la consolidación de EBT inteligentes.

\section{Aprendizaje en el proceso de incubación de EBT en Brasil}

Autores como Cassiolato \& Lastres (1999) y Albuquerque (2003), caracterizaron el 
Sistema Nacional de Innovación brasileño como incompleto y precario debido a tres factores: 1) un sistema de producción basado inicialmente en la gran empresa, el cual es excesivamente exocéntrico, dependiente de capital y de tecnología de origen externo, lo que dificulta el proceso de aprendizaje, 2) debilidad financiera y tecnológica y 3) pulverización sectorial de las pequeñas empresas y débiles interacciones de los actores dentro del sistema productivo.

Estas características dificultan y reducen las posibilidades del aprendizaje por interacción, elemento esencial del aprendizaje en sí. No obstante, recientemente Nader (2013), ha señalado que en Brasil luego de más de seis décadas, el SNI es altamente sinérgico y organizado, lo que ha permitido producir conocimiento reconocido internacionalmente, llevando a que el país haya logrado ocupar el décimo tercer lugar en el ranking de la producción científica mundial; empero, también se identifica la existencia de grandes amenazas para el sector de ciencia y tecnología relacionadas con intereses políticos y disminución de la financiación.

Maculan \& Carvalho de Mello (2009), afirman que desde la década de 1990, el modelo de desarrollo económico de Brasil ha enfatizado una mayor eficiencia en la gestión y la innovación, puesto que como ocurrió en otros países de Latinoamérica en dicha época, cambios como la privatización y la reducción de barreras comerciales enfrentaron a las empresas brasileñas a la competencia internacional sin eficiencia en la gestión ni suficientes capacidades tecnológicas. El gobierno dio un decidido apoyo e incentivó la relación universidad - empresa y se estableció un conjunto de actividades de interacción, lideradas por las universidades fuertes en I+D y con incubadoras de base tecnológica, lo que permitió entre otros, que la cantidad de estas incubadoras aumentara notablemente durante esta década en muchas universidades públicas del país.
El surgimiento de las incubadoras de empresas en Brasil es un factor importante en la formación de un ambiente institucional favorable al desarrollo del emprendimiento y al proceso de aprendizaje de las EBT, como un proceso fuertemente vinculado al ámbito económico local, representando una experiencia original y característica de la historia de la industrialización brasileña. (Maculan, 2003)

Las incubadoras de empresas son organizaciones cada vez más populares que se crean, a menudo con la ayuda de las agencias de desarrollo económico, para apoyar y acelerar el avance y éxito de las empresas afiliadas (Hansen, Chesbrough, Nohria, \& Sull, 2000). De acuerdo con McAdam \& McAdam (2008), en el caso de las incubadoras universitarias, la cooperación con los empleados de la universidad permite el acceso al conocimiento más reciente en el área de interés, resultando en el desarrollo de productos más innovadores; así mismo, el vínculo con la universidad puede permitir una reducción en los costos de desarrollo de productos, dando al cliente la garantía de que están basados en el conocimiento más nuevo disponible, mientras que las habilidades especializadas que poseen los estudiantes pueden ser aprovechadas por las empresas dada la cercanía de la incubadora a la universidad.

En el año 2009 se creó el Centro de Referencia para el Apoyo a Nuevos Emprendimientos Cerne por iniciativa de la Asociación Nacional de Entidades Promotoras de Emprendimientos Innovadores Anprotec y el Servicio Brasileño de Apoyo a las Micro y Pequeñas Empresas Sebrae, con el objetivo de promover mejores resultados en las incubadoras de empresas, identificando sistemas, elementos y prácticas clave requeridos en una incubadora para lograr la generación sistemática de cantidades cada vez mayores de emprendimientos innovadores exitosos; el abordaje de Cerne 
se da en los niveles de empresa, proceso de incubación e incubadora. Cerne (citado por Iacono \& Seido, 2014)

Las incubadoras en Brasil son instituciones creadas en su mayoría por iniciativa de las universidades, apoyadas por las secretarías municipales, los centros estatales de ciencia y tecnología o las agencias de apoyo a la investigación, para abrigar a empresas que nacen de proyectos innovadores (Baeta \& Maculan, 1995; Lemos \& Maculan, 1998). Las incubadoras de base tecnológica corresponden al $40 \%$ del total de incubadoras en el país, y en su mayoría operan apoyando iniciativas de universidades y centros de investigación que se consolidan como spin-offs (Anprotec, 2011). Ellas ofrecen un espacio institucional de apoyo para tales empresas, las cuales aprenderán, en tres o cuatro años, a actuar en el mercado como organizaciones productivas plenas. Ver Figura 2.

\section{Características del proceso de aprendizaje de acuerdo con la evidencia empírica}

Los elementos analizados a continuación fueron obtenidos en diversos estudios de campo realizados en Brasil (Maculan, 1996; Iacono \& Seido, 2014; Maculan, 2003; Pereira \& Maculan, 2001). La relativamente corta experiencia brasileña en creación de empresas a través de las incubadoras, hace que las situaciones empíricas sean extremamente diversas y que las empresas presenten desempeños desiguales.

En los estudios referenciados se encontró que las empresas que se implantan en las incubadoras de las universidades brasileñas son necesariamente de base tecnológica, ya que esta característica hace parte del proceso de selección de las ideas

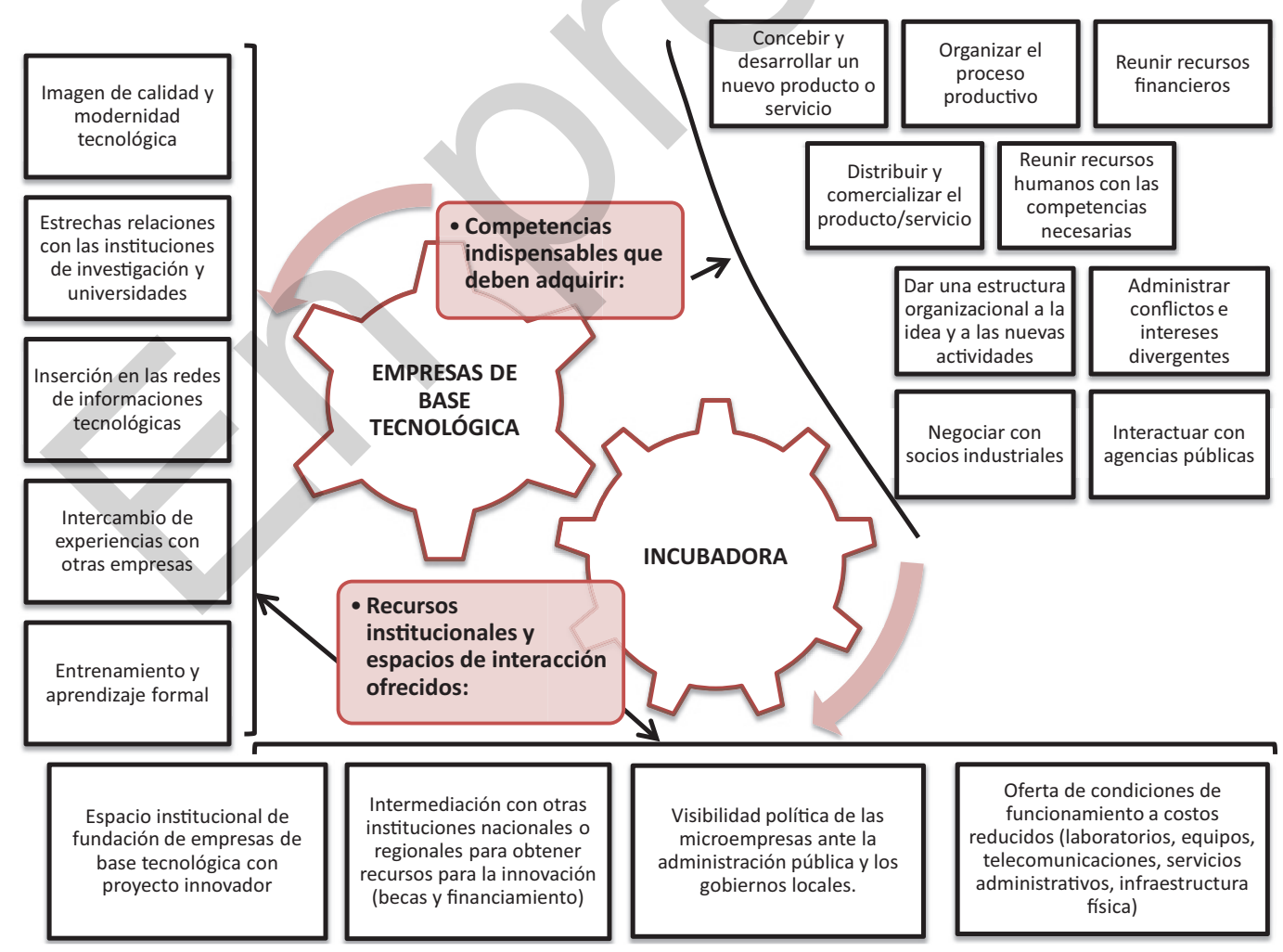

Figura 2. Recursos que ofrecen las incubadoras y competencias que generan las empresas incubadas 
de negocio que pretenden beneficiarse de los servicios ofrecidos por dichas incubadoras. El período de incubación es en esencia un período de aprendizaje ya que los nuevos empresarios raramente poseen experiencia anterior en gestión (los fundadores de empresas en las incubadoras frecuentemente son estudiantes recién graduados en ingeniería, matemática o informática, o de postgrado).

El aprendizaje puede ser organizado de manera formal por la incubadora a través de cursos de mercadeo o de oferta de asesorías específicas, pero también existe un proceso de aprendizaje informal y tácito, basado en el intercambio de experiencias con otras empresas de la incubadora, en las orientaciones recibidas de los gerentes, en las experiencias del tipo ensayo y error, en el trabajo de identificación de proveedores y compradores, en la búsqueda de informaciones técnicas con los investigadores de la universidad, en la realización de pruebas de productos con los usuarios, en la identificación de posibles socios para ampliar la base financiera de la empresa, en la prestación de servicios para obtener recursos en ausencia de un producto desarrollado en su totalidad, entre otros.

Durante la permanencia en la incubadora, los empresarios pueden buscar, incorporar, asimilar y acumular conocimientos, conseguir informaciones, adoptar nuevos comportamientos, organizar la producción, reunir recursos humanos, técnicos, administrativos y financieros. Requieren identificar los socios (proveedores y clientes) y establecer relaciones de negocios con ellos. El alto nivel de educación de estos empresarios facilita el aprendizaje, las interacciones y la innovación. En su mayoría, están familiarizados con las actividades de investigación y se muestran capaces de aprender a través de la búsqueda (learning by searching).

$\mathrm{Al}$ analizar estas empresas, se constata la existencia de múltiples interacciones con los responsables de la incubadora, otros empresarios, los proveedores, los clientes o los usuarios y las instituciones de investigación y de enseñanza (existe gran cantidad de intercambios con ex-profesores o ex-compañeros de estudios que son requeridos para ayudar en el desarrollo de productos de la nueva empresa). No obstante, se ha evidenciado que las relaciones con clientes son de baja intensidad respecto al desarrollo de nuevos conocimientos.

La incubadora funciona como un intermediario indispensable para reducir los costos de acceso a las informaciones de naturaleza legal o técnica y a los recursos materiales, humanos y financieros (pedidos de becas para obtener recursos humanos calificados, negociación con las entidades de apoyo, solicitudes de financiamiento ante las agencias gubernamentales para la innovación). Ella permite las interacciones con un mayor número de organizaciones y favorece a la creación de un capital intangible de conocimientos.

De esta manera, las empresas se insertan en las redes de oferta de productos y servicios, adquieren paulatinamente una experiencia organizacional para desarrollar, producir, financiar, comercializar y distribuir sus productos y aprenden a definir sus procedimientos construyendo una memoria activa de resolución de problemas técnicos, financieros o gerenciales. Las empresas incubadas logran generar un clima organizacional que facilita el trabajo en equipo y la socialización de ideas y sugerencias para mejorar procesos o productos, si bien la toma de decisiones es todavía más intuitiva que analítica. 
En general, se observan buenos resultados de los procesos de aprendizaje, en los cuales se destacan actividades relativas a la formación y capacitación del personal; sin embargo, el aprendizaje se tropieza con ciertos comportamientos: la predominancia de la competencia técnica sobre la gerencial, la sobrevalorización de la figura del emprendedor como individuo, la escasez o la movilidad de los recursos humanos (becarios o practicantes universitarios). Así mismo, son pocas las empresas que se comparan de forma sistemática con sus competidores como medio de aprendizaje; el desempeño y las experiencias de las otras empresas incubadas o del gerente de la incubadora pueden facilitar o no el aprendizaje debido a la importancia del efecto de demostración.

\section{Papel de las incubadoras en el proceso de aprendizaje de las EBT}

Un criterio para evaluar la eficiencia del aprendizaje por parte de las EBT será, al final, la capacidad de cada empresa de salir de la incubadora, establecerse de forma autónoma y permanecer en el mercado. Si las empresas están más tiempo en la incubadora, se puede concluir que existe, de hecho, un proceso de aprendizaje más eficiente que el que ocurre fuera de ella. Esta es la primera prueba de la importancia de la dimensión institucional del aprendizaje y es el principal motivo para la adopción de políticas de apoyo a la implementación de incubadoras en Brasil. La "graduación" de las nuevas empresas es una forma de comprobar a posteriori un aprendizaje exitoso de búsqueda de conocimientos y de desarrollo de formas institucionales para el manejo de conflictos e intereses divergentes; pero permanece una gran diversidad de desempeño y de capacidad de aprendizaje.
Otros factores son esenciales para el proceso de aprendizaje de las EBT incubadas en Brasil: la visibilidad institucional de la incubadora y las experiencias de otras empresas incubadas. La habilidad para movilizar los recursos políticos, financieros y organizacionales y la experiencia administrativa de los gerentes de las incubadoras son muy diversas, pero tienen una gran importancia en el proceso de aprendizaje organizacional de las empresas, la inserción en las redes de informaciones y en última instancia, en la definición de la estrategia y el desempeño en el mercado de las empresas incubadas. Las habilidades gerenciales de la incubadora determinan la calidad del aprendizaje organizacional y de las interacciones desarrolladas por las EBT.

Cada incubadora detiene un conjunto de competencias que en parte condiciona la rapidez, el contenido y la eficacia del aprendizaje de las empresas a partir de la oferta de interacciones con otras instituciones. Al respecto Iacono \& Seido (2014), encontraron que los emprendedores valoran principalmente el acompañamiento empresarial y gerencial ofrecido por las incubadoras, así como la consultoría y asesoría en negocios, teniendo en cuenta que en su mayoría son personas que por primera vez realizan un emprendimiento de base tecnológica; por otra parte, la consultoría y asesoría técnica especializada generan un menor grado de satisfacción, dado que su aporte ocurre principalmente al inicio de la incubación, pero en etapas posteriores las necesidades de las empresas son otras; en la Tabla 1 se presentan los aspectos no encontrados en las incubadoras por parte de los empresarios brasileños consultados en la investigación de estos autores. 
Tabla 1.

Recursos clave para los empresarios que generalmente no ofrecen las incubadoras

\begin{tabular}{cl}
\hline Áreas o temas & \multicolumn{1}{c}{ Recursos } \\
\hline $\begin{array}{c}\text { Gestión } \\
\text { financiera }\end{array}$ & Acceso a fondos de inversión y capital de riesgo \\
Acompañamiento permanente en eventuales aportes de capital de riesgo \\
\hline $\begin{array}{c}\text { Gestión } \\
\text { empresarial }\end{array}$ & Acompañamiento más frecuente a los procesos de desarrollo del negocio \\
Mejor orientación en las consultorías sobre temas tributarios y laborales. \\
\hline $\begin{array}{c}\text { Mercadeo y } \\
\text { comercialización }\end{array}$ & Orientación en los procesos de homologación \\
& Actuar como facilitadora junto a los órganos de regulación \\
Aercadeo de & Ser facilitadora en las relaciones con la universidad \\
relacionamientos & Ofrecer más redes de contactos con otras empresas e instituciones de apoyo \\
\hline Tecnología e & Mejor orientación en temas de innovación \\
Consultorías en tecnología \\
Pruebas de laboratorio y montaje de prototipos \\
\hline
\end{tabular}

Fuente: Iacono \& Seido (2014)

La evaluación de la eficacia del aprendizaje ocurre en el momento en que la empresa se muestra capaz de establecerse en el mercado de manera autónoma. El principal argumento a favor de las incubadoras es constatar que el porcentaje de mortalidad de las empresas es menor en aquellas que tuvieron un proceso de incubación. Está claro que esa diferencia viene dada por las condiciones más favorables y los procesos de aprendizaje mejores y más diversos. Al dejar la incubadora, la EBT muestra que el aprendizaje fue exitoso.

\section{Grado de asimilación del conocimiento y la tecnología en las EBT de Brasil}

Tomando en cuenta la categorización propuesta por Granados et al. (2006), las EBT incubadas en Brasil se pueden considerar en su mayoría como tecnicistas, ya que su dominio del conocimiento tecnológico es alto, al estar integradas por empresarios con alta formación académica y experticia en el área de la tecnología que sirve de base al proceso productivo, pero con escasa experiencia en gestión, lo que dificulta la promoción de procesos de aprendizaje organizacional.

Se puede identificar un dinamismo tecnológico en las EBT incubadas, relacionado con la necesidad de monitorear los avances de conocimiento en el contexto mundial, lo que les permite entender mejor su base tecnológica y lograr la integración de estos nuevos conocimientos en sus actividades de producción.

A pesar de que las EBT incubadas tienen una base amplia de conocimiento tecnológico requerido para la creación de la empresa, continúa la exigencia de un mayor aprendizaje respecto al desarrollo y colocación de productos en el mercado, puesto que los problemas y retos técnicos aún no están resueltos, y la necesidad de avance permanece. La EBT debe considerar que cualquier producto nuevo no es satisfactorio, y que debe contemplar el servicio al cliente, así como la atención de problemas del usuario y del producto en sí. 
De acuerdo con Granados et al. (2006), las EBT tecnicistas deben diseñar y realizar procesos de cambio cultural, adoptar el paradigma de la organización que aprende y modelos de gestión del conocimiento tendientes a propiciar el aprendizaje organizacional, el trabajo en equipo, a mejorar la comunicación entre sus integrantes como elementos clave en la generación de conocimiento propio de toda la organización y en la elaboración de productos y servicios competitivos.

Las empresas Inteligentes son vistas como las más competitivas en el contexto de la sociedad del conocimiento y por tanto, las de mayor sostenibilidad en el tiempo. En la búsqueda de una transición de las EBT incubadas en Brasil, desde el tecnicismo hacia la inteligencia, cualquier modelo de gestión que se adopte no será suficiente, ya que se requieren cambios en el ámbito organizacional, tecnológico, cultural, entre otros.

En este proceso de transición hacia las empresas inteligentes, las EBT incubadas en Brasil tienen una gran ventaja frente a aquellas que no experimentan el proceso de la incubación, puesto que la incubadora ofrece asesoría y capacitación en temas diversos, dando herramientas a los empresarios para incentivar el aprendizaje organizacional de forma simultánea con el fortalecimiento de su base tecnológica, y contribuyendo a la generación de competencias gerenciales y tecnológicas. Adicionalmente, cuando se trata de incubadoras universitarias, este acercamiento facilita el acceso al conocimiento de punta.

Las EBT son empresas pequeñas, que por contar con recursos limitados, requieren establecer contactos con otras organizaciones como base para la colaboración en búsqueda de mejoras en sus procesos de producción, comercialización y distribución con miras a su inserción en redes. En el caso de las empresas incubadas en
Brasil, el papel de las incubadoras ha sido clave para avanzar en dicha vinculación y crear bases para su capital relacional, que deberá fortalecerse por cuenta de la empresa cuando salga de la incubadora.

\section{CONCLUSIONES}

Al analizar las empresas de base tecnológica incubadas en Brasil es posible caracterizar las instancias e interacciones que activan los procesos del aprendizaje. El contenido y la intensidad del aprendizaje determinan la inserción dinámica de estas empresas en el sistema nacional de producción de Brasil, con un importante papel para la formación de un verdadero Sistema Nacional de Innovación que aún no ha sido completado.

La localización en las incubadoras posibilita la inserción de las EBT en redes de información, producción y servicios, que incluso pueden surgir como instituciones informales (conexiones de redes interpersonales y contactos generados en el quehacer de la incubadora), para funciones específicas como obtención de información del mercado o interpretación de normas, en respuesta a una posible debilidad de las instituciones formales representadas en leyes o normatividad de fomento a las incubadoras. (Moreno \& Ramos, 2013)

Tal inserción es un factor esencial para el establecimiento de rutinas y la adquisición de competencias productivas y organizacionales. Las empresas que son capaces de entrar en las redes de cooperación consiguen: 1) aprender y ampliar el saber acumulado, 2) adquirir capacidad de adaptación a los cambios e 3) innovar $y$ construir ventajas competitivas. El éxito de la trayectoria de las EBT incubadas está fuertemente determinado por la capacidad de la incubadora para activar diversas instituciones del Sistema de Innovación, sea en el nivel regional o nacional. 
Puede afirmarse que la localización de las EBT en las incubadoras posibilita la adquisición de competencias productivas organizacionales, la inserción en diversas redes y la considerable reducción de los riesgos y costos inherentes a su establecimiento en el mercado. El desafio más importante para las EBT es salir de la incubadora, lo que significará que fueron capaces de concebir un producto o un nuevo servicio, organizar el proceso productivo, manejar conflictos, atraer recursos humanos calificados y definir una estrategia en el mercado. No obstante, algunas dificultades permanecen como la poca visibilidad en el mercado y la imagen negativa de la inexperiencia.

La transición de las EBT desde la categoría tecnicista a la categoría de empresas inteligentes es un proceso lento y difícil, que requiere gran inversión en recursos y la generación de capacidades para la búsqueda de los mismos, así como el desarrollo de una visión estratégica y competencias gerenciales. La localización de las empresas nacientes dentro de una incubadora favorece esta transición, por la capacidad y recursos con que cuenta la entidad de incubación, que le permite ofrecer un complejo y estructurado sistema de apoyo a los emprendedores, poniéndolos en ventaja frente a aquellos que no experimentan el proceso de incubación.

\section{REFERENCIAS}

Albuquerque, E. (2003). Immature systems of innovation: Introductory notes about a comparison between South Africa, India, Mexico and Brazil based on science and technology statistics. Belo Horizonte: Cedeplar, Universidade Federal de Minas Gerais.
Anprotec, (2011). Incubadoras e Parques. Recuperado de: http://anprotec.org.br/site/pt/incubadoras-eparques/

Arocena, R. \& Sutz, J. (2006). El estudio de la Innovación desde el Sur y las perspectivas de un Nuevo Desarrollo. CTS+I: Revista Iberoamericana de Ciencia, Tecnología, Sociedad e Innovación, (7), Recuperado de: www.oei.es/revistactsi/numero7/articulo01.htm

Baeta, A. M. C. \& Maculan, A. M. (1995). Uma nova perspectiva organizacional: as incubadoras de empresas de base tecnológica. $X X X$ Assembléia do CLADEA. "Administração de Serviços” São Paulo: FEA/USP.

Bell, M. (1984). Learning and the Accumulation of Industrial Technological Capacity in Developing Countries. In M. Fransman \& K. King (Eds.), Technological capability in the Third World, 187-209, Basingstoke, UK: Macmillan Press.

Carvalho, M. M., Machado, S., Pisysieznig-Filho, J. \& Rabechini-Junior, R. (1998). Empresa de Base Tecnológica Brasileira: características distintivas. XX Simpósio de Gestão da Inovação Tecnológica, 20, 461-474.

Cassiolato, J. E. \& Lastres, H. M. M. (1999). Local, national and regional systems of innovation in the Mercosur. DRUID's Summer Conference on National Innovation Systems, Industrial Dynamics and Innovation Policy. Denmark: Danish Research Unit on Industrial Dynamics.

Castellanos, O. (2007). Gestión Tecnológica: de un enfoque tradicional a la inteligencia. Bogotá: Editorial Universidad Nacional de Colombia. 
Chamanski, A. \& Waagø, S. J. (2001). The organizational success of new, technology-based firms. working paper. Trondheim: Norwegian University of Science and Technology.

Clarysse, B. \& Moray, N. (2004). A process study of entrepreneurial team formation: the case of a researchbased spin-off. Journal of Business Venturing, 19(1), 55-79. doi: 10.1016/ S0883-9026(02)00113-1

Cooke, P., Gomez, M. \& Etxebarria, G. (1997). Regional innovation systems: Institutional and organisational dimensions. Research Policy, 26(4), 475-491. doi: 10.1016/S00487333(97)00025-5

Dutrénit, G. (2001). El papel de las rutinas en la codificación del conocimiento en la firma. Análisis Económico, 16(34), 211-230.

Estrada, S. (2005). Economía de la innovación en el contexto mexicano. Programa Semanal de Seminarios de la Escuela de Economía, 11. Recuperado de: www.fimee.ugto.mx/profesores/salvadorer/documentos/IDTcap\%201_exp_prof_Economia\%20 de\%20la\%20innovacion.doc

Granados, O., Castellanos, O. \& León, A. (2006). Incidencia del nivel de asimilación del conocimiento y la tecnología en el desarrollo empresarial. X Congreso anual de la Academia de Ciencias Administrativas México: ACACIA.

Hansen, M. T., Chesbrough, H. W., Nohria, N. \& Sull, D. N. (2000). Networked incubators. Harvard business review, 78(5), 74-84.

Hung, R. Y. Y., Lien, B. Y.-H., Yang, B., Wu, C. M. \& Kuo, Y. M. (2011). Impact of TQM and organizatio- nal learning on innovation performance in the high-tech industry. International Business Review, 20(2), 213-225. doi: 10.1016/j.ibusrev.2010.07.001

Iacono, A. \& Seido, M. (2014). Gestão da inovação em empresas nascentes de base tecnológica: Evidências em uma incubadora de empresas no Brasil. Interciencia, 39(5), 296-306.

Incubadora de Empresas de Base Tecnológica de Antioquia - IEBTA. (2006). Estrategia corporativa de la Incubadora de Empresas de Base Tecnológica de Antioquia. Incubadora de Empresas de Base Tecnológica de Antioquia. Recuperado de: www.incubadora.org.co

Jiménez, C. N. \& Castellanos, O. (2008). Retos de la gestión tecnológica para el siglo XXI. In Retos y nuevos enfoques en gestión de la tecnología y del conocimiento. Bogotá, Colombia: Giro Editores.

Jiménez, D. \& Sanz, R. (2006). Innovación, aprendizaje organizativo y resultados empresariales: un estudio empírico. Cuadernos de Economía y Dirección de la Empresa, (29), 3155 .

Katz, J. (1984). Technological innovation, industrial organization and comparative advantages of Latin American metalworking industries. In M. Fransman \& K. King (Eds.), (pp. 113-136). Basingstoke, UK: Macmillan Press.

Lemos, M. V. \& Maculan, A. M. (1998). $\mathrm{O}$ papel das incubadoras no apoio às empresas de base tecnológica. $X X$ Simpósio de Gestão da Inovação Tecnológica. São Paulo, Brasil: NPGCT - IA - USP. 
Lundvall, B. A. (1988). Innovation as an interactive process: from userproducer interaction to the national system of innovation. In G. Dosi, C. Freeman, R. Nelson, G. Silverberg, \& L. Soete (Eds.), Technical Change and Economic Theory. London: Pinter Publishers.

Maculan, A. M. (1996). From research to innovation: the Brazilian experience with business incubators. Londres, Nueva York: Printer.

Maculan, A. M. (2003). Ambiente empreendedor e aprendizado das pequenas empresas de base tecnológica. Rio de Janeiro: Relume Dumará.

Maculan, A. M. \& Carvalho de Mello, J. M. (2009). University start-ups for breaking lock-ins of the Brazilian economy. Science and $\mathrm{Pu}$ blic Policy, 36(2), 109-114. doi: 10.3152/030234209X406791

McAdam, M. \& McAdam, R. (2008). High tech start-ups in University Science Park incubators: The relationship between the start-up's lifecycle progression and use of the incubator's resources. Technovation, 28(5), 277-290. doi: 10.1016/j. technovation.2007.07.012

Morales, M. E., Castellanos, O. \& Jiménez, C. N. (2007). Consideraciones metodológicas para el análisis de la competitividad en empresas de base tecnológica. Revista Facultad de Ciencias Económicas: Investigación y Reflexión, 15(2), 97-112.

Morales, S. (2009). Una Aproximación a las Empresas Basadas en Investigación (Spin - Off). Medellín.

Moreno, J. \& Ramos, J. (2013). Factores determinantes de la creación de empresas de base tecnológica en Colombia. Revista de Economía del Caribe, (19), 1-29.

Mustar, P., Clarysse, B. \& Wright, M. (2007). University spin-off firms in Europe: What have we learnt from ten years experience? Policies for Research and Innovation in the Move Towards the European Research Area: Vol. 29. PRIME 3rd annual Conference Pisa, Italia.

Nader, H. (2013). A preservação da ciência e tecnologia. Folha de S.Paulo. Recuperado de: www.folha.uol.com. br/opiniao/1213098-tendenciasdebates-a-preservacao-da-ciencia -e-tecnologia.shtml

Nelson, R. (2003). Insertar las instituciones en la teoría evolutiva del crecimiento. Análisis Económico, 18(38), 123-138.

Nelson, R. R. \& Winter, S. G. (1982). An evolutionary theory of economic change. Cambridge: Harvard University Press.

Pereira, M. G. \& Maculan, A. M. (2001). Empresas graduadas nas incubadoras brasileiras. Brasilia: CT Brasil. MCT/SEPTE. IEL.

Rasmussen, E. \& Borch, O. J. (2010). University capabilities in facilitating entrepreneurship: A longitudinal study of spin-off ventures at mid-range universities. Research Policy, 39(5), 602-612. doi: 10.1016/j.respol.2010.02.002

Simón, E. K. (2003). La creación de empresas de base tecnológica, una experiencia práctica. Proyecto para la promoción de empresas innovadoras de base tecnológica. España, Ministerio de Ciencia y Tecnología. 


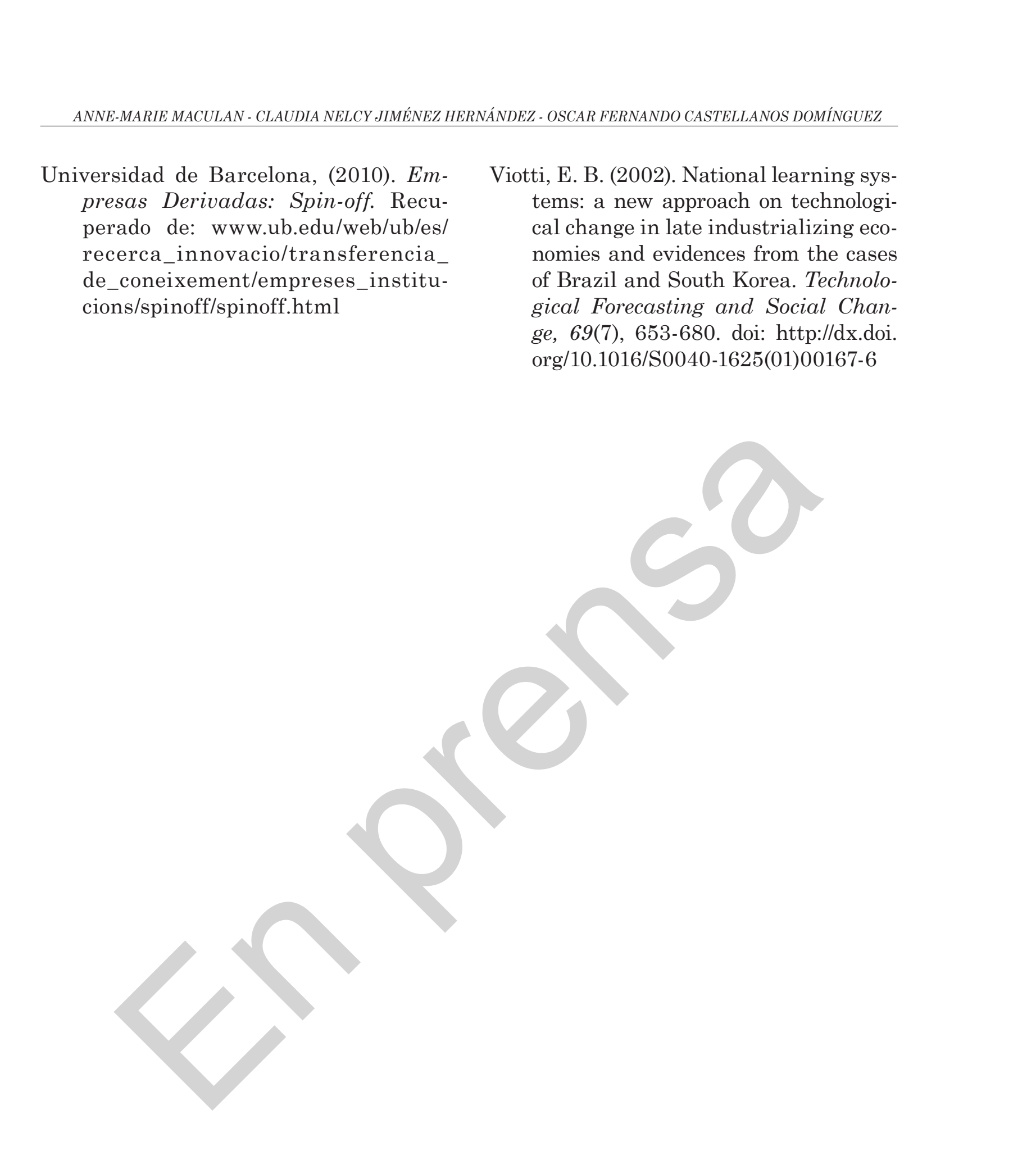

